\title{
The Practice of Showing 'Who I am': A Multimodal Analysis of Encounters between Science Communicator and Visitors at Science Museum
}

\author{
Mayumi Bono ${ }^{1,2}$, Hiroaki Ogata ${ }^{3}$, Katsuya Takanashi ${ }^{4}$, and Ayami Joh ${ }^{1}$ \\ ${ }^{1}$ Digital Content and Media Sciences Research Division, National Institute of Informatics \\ ${ }^{2}$ Department of Informatics, School of Multidisciplinary Sciences, \\ The Graduate University for Advanced Studies (SOKENDAI) \\ 2-1-2 Hitotsubashi, Chiyoda-ku, Tokyo 101-8430, Japan \\ \{bono, joh\}@nii.ac.jp \\ ${ }^{3}$ Graduate School of Information Science and Electrical Engineering, Kyushu University \\ 744, Motooa, Shishi-ku, Fukuoka 819-0395, Japan \\ hiroaki.ogata@gmail.com \\ ${ }^{4}$ Academic Center for Computing and Media Studies, Kyoto University \\ Yoshida-Honmachi, Sakyo-ku, Kyoto 606-8501, Japan \\ takanasi@ar.media.kyoto-u.ac.jp
}

\begin{abstract}
In this paper, we try to contribute to the design of future technologies used in science museums where there is no explicit, pre-determined relationship regarding knowledge between Science Communicators (SCs) and visitors. We illustrate the practice of interaction between them, especially focusing on social encounter. Starting in October 2012, we conducted a field study at the National Museum of Emerging Science and Innovation (Miraikan) in Japan. Based on multimodal analysis, we examine various activities, focusing on how expert SCs communicate about science: how they begin interactions with visitors, how they maintain them, and how they conclude them.
\end{abstract}

Keywords: Multimodal Interaction Analysis, Social encounter, Science Communicators (SCs), Science Museum.

\section{Introduction: Visiting the Science Museum}

When we visit a medical clinic, we typically have a clear reason to go there, for example, we have a fever or stomachache, and so forth. The doctor has specific knowledge, skills, and techniques to cure those diseases for the patient. According to the concept of a membership categorization device proposed by Sacks (1974), the relationship between doctor and patient is explicit, i.e., the doctor is a plus $K$ person, a professional, and the patient is a minus $K$ person, a nonprofessional. Furthermore, people do not visit a medical clinic unless they have some kind of health question or issue. Thus, the clinic is an institutional setting in which the purposes of the activities, the roles of the participants, and the direction of the provision of specific knowledge are almost pre-determined (Drew \& Heritage, 1992). 
On the other hand, science museums are sometimes ambiguous spaces. Some visitors have a concrete motivation or intention when they visit museums, such as learning about current trends in science and technology, learning about Newton's theory of universal gravitation, and so on. However, many people visit museums on school trips, which means that the individuals do not have the clear intention to visit them. They do not necessarily understand in advance what kinds of scientific things they will learn at the museums.

Some science museums in Japan, for instance the National Museum of Emerging Science and Innovation (hereafter Miraikan) and the National Museum of Nature and Science in Japan, have established Science Communicators (hereafter SCs) to share their knowledge of science with people visiting the museum. However, people who visit the museum as a part of school trip usually do not know about the existence or even the concept of SCs. Thus, we assume that there is no explicit relationship between SCs and museum visitors who are interested in scientific knowledge.

In this paper, we try to contribute to the design of future technologies used in science museums where there is no explicit, pre-determined relationship regarding knowledge between SCs and visitors and we illustrate the practice of interaction between them, especially focusing on social encounter.

\section{Background: What 'SC' Is}

Starting in October 2012, we conducted a field study at Miraikan in Japan. In April 2012, before we started our fieldwork, one of the science communicators, who had 3 years' experience as a SC at that time, gave us the opportunity to observe and examine their daily activities at Miraikan. He had been trying to establish criteria by which to evaluate the skills of science communicators and to build an education system for better communication between SCs and visitors before his projected departure from Miraikan in March 2014.

Almost all of the SCs at Miraikan have 5-year employment contracts. They were selected from various academic fields, and some SCs hold masters or doctoral degrees. The SC who explained this position to us also works under this contract, and he had a long academic career before coming to Miraikan. During these 5 years, SCs learn communication skills intended to interest the public in science and to entertain them. They meet visitors directly on the exhibition floor.

When SCs describe their activities at Miraikan to outsiders, they always say that there is a clear boundary between science communicators and interpreters or curators in a museum. Even if they start to explain an exhibition to visitors, they explain that expert SCs are able to change the exchange from a straightforward explanation to a dialogue.

SCs at Miraikan mainly have three kinds of activities inside the museum: (1) they have a booth where they sit to explain specific research themes using model kits of objects such as the brain; (2) they sometimes present mini-lectures in a lecture hall, explaining specific knowledge regarding science based on their own experience; and 
(3) each of them has time in which to interact with visitors on the exhibition floor for a pre-determined 1-hour shift.

In this paper, we examine the third of these activities practiced by SCs, focusing on how expert SCs communicate about science: how they begin interactions with visitors, how they maintain them, and how they conclude them, based on multimodal analysis.

\subsection{Repository of Skills in the Oral Tradition}

This research project is a collaborative work with the practitioners, namely SCs. One of the aims of this project is to detect the features of these interactions that SCs believe contribute most significantly to effective science communication based on multimodal interaction studies and conversation analysis (CA) of SCs' work in the field. When we asked expert SCs what kind of interactions are suitable and ideal as science communication, showing video clips that we filmed, their answers, amazingly, coincided. It seems that some of the expert SCs have already established common ways and standards by which to evaluate SC skills. Based on these shared standards among the expert SCs, criteria to evaluate trainee SCs and manuals for teaching science communication methods were developed at this museum.

However, from the perspective of observers, we suspect that it is impossible to create definitive criteria and write a standard manual for this field. This is related to the issue of the ambiguity of visitors' intentions and the amount of knowledge transferred between SCs and visitors, mentioned in the previous section. If visitors have clear intentions and if an epistemic difference (Heritage, 2012) between those involved in the interaction is apparent, it is easy for SCs to understand what kinds of actions they need to take in terms of specific interactions, which makes it easier to develop a manual about how to better interact with visitors.

Through observing their daily activities for a year, we realized that, in fact, aspects of science communication are rooted in the oral tradition. The oral tradition is a Japanese-specific way in which professionals hand their special skills on to the next generation, e.g., traditional arts, crafts, and performances. We assume that SCs at Miraikan have unintentionally chosen this Japanese-specific way in which to share their special skills and knowledge for better science communication. This is in spite of the fact that the SC position was part of a top-down program developed and implemented by the ministry of Science and Technology in Japan as a modern and scientific system by which to share knowledge with the public. During this transitional period, as they work to complete the task of creating criteria and preparing a manual that identifies scientific ways to share their skills and knowledge, we attempt to illuminate how the repository of skills of expert SCs is shared within the SC community.

\subsection{Video Recording and Reflexive Anthropology}

Using recording technology, we established an environment that enabled us to observe a targeted phenomenon over and over again (Heath, Hindmarsh, Luff, 2010). 
As we mentioned before, because SCs have their own views and opinions regarding the practice of science communication, we prepared a place where SCs and interaction analysts could discuss the concrete purposes of their actions and their alignment with the traditional understandings of conversation analysis (CA) related to the following: how SCs initiate conversational sequences in semi-open spaces such as museums; how SCs invite visitors to participate in a scientific dialogue; and how SCs manage their time during interactions with visitors and explain their choices during conversations with other SCs, which we refer to as 'reflection meetings' in this paper. We assumed that we could use the practitioners' voices at these meetings, selecting crucial segments from their practices to illuminate how expert SCs evaluate the communication skills of other SCs.

To this end, we decided to film the embodied actions of SCs not only with visitors but also at a reflection meeting held after closing to discuss the events that occurred on the day. We expected to obtain input from the SCs about their own skills (e.g., how they evaluate their skills and share experiences about similar cases) during this meeting. This paper adopts the perspective of CA to discuss how we can use the knowledge of SCs to analyze their interactions with visitors. The reflection meeting provided information about the current SCs' impressions of events and visitors, such as why they chose to engage in a particular action with visitors at a given time. Additionally, we also obtained advice from senior SCs regarding what can be done to include visitors in science communication. It is impossible to obtain this type of information about the behaviors of SCs using only a traditional transcription system. In other words, the current CA system is unable to elicit information about the impressions of the interacting individuals and to solicit advice from key individuals. These shortcomings preclude our understanding the personal observations that lead to particular organizations of sequences and potential next steps. We discuss the boundary between the resources that can and cannot be involved in CA.

\section{The Data}

The data collection was divisible into three phases according to the recording devices used and the purpose of filming. For the first phase, we mainly conducted research using a professional high-definition camera and a professional microphone (see Fig.1) for filming the practices of two expert SCs (7 and 3 years' experience, respectively), one medium-level SC (1 year's experience), and two novice SCs (1 month's experience) from October to November 2012. After each filming session, we convened a reflection meeting, held within 1 or 2 hours after SCs finished their interactions with visitors on the floor (see Fig.2). We filmed the meeting at which SCs and conversation analysts discussed current SCs' impressions of events and visitors, such as why they chose to engage in a particular action with visitors at a given time. Additionally, we also obtained advice from senior SCs regarding what could be done to include visitors in science communication. This filming was conducted under the supervision of the ethics board of National Institute of Informatics, Japan. 
Before the filming, we determined the permanent exhibition at which we would collect data, deciding on an exhibition on the third floor of the Miraikan that focused on future technologies. This exhibit, called 'The Driving Force of Innovation' consists of three elements (see Fig.3). The first element, 'The Spring of Wishes,' was designed so that past visitors' written wishes concerning technology come up from the central hall spinning in a circle triggered by current visitors when they put a ball into a slot outside. The second element, 'The River of Creativity,' was designed so that each of five rivers has one scientific question written Japanese and one interactive model for learning about scientific innovations. These rivers reach 'The Sea of Fertility' at the end, which is just a blackboard on which visitors can write down their ideas about society in 2020 in relation to science and technology. It is titled 'Let's think about our society in 2020,' which is written at the top of the blackboard, and it has magnets representing 'seeds of technology.' This is where visitors come at the end of the exhibition, a place where they can write or draw their ideas for the future using magnets.

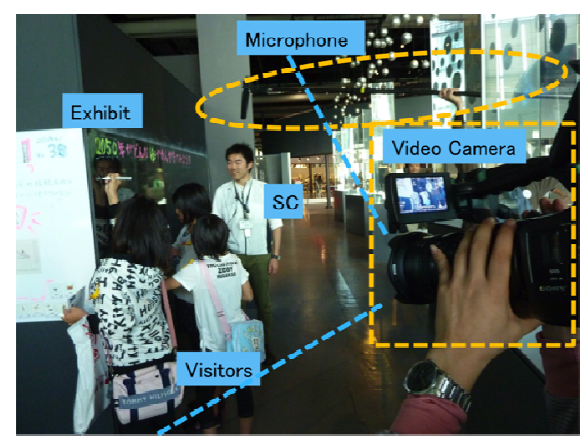

Fig. 1. Filming during the first phase

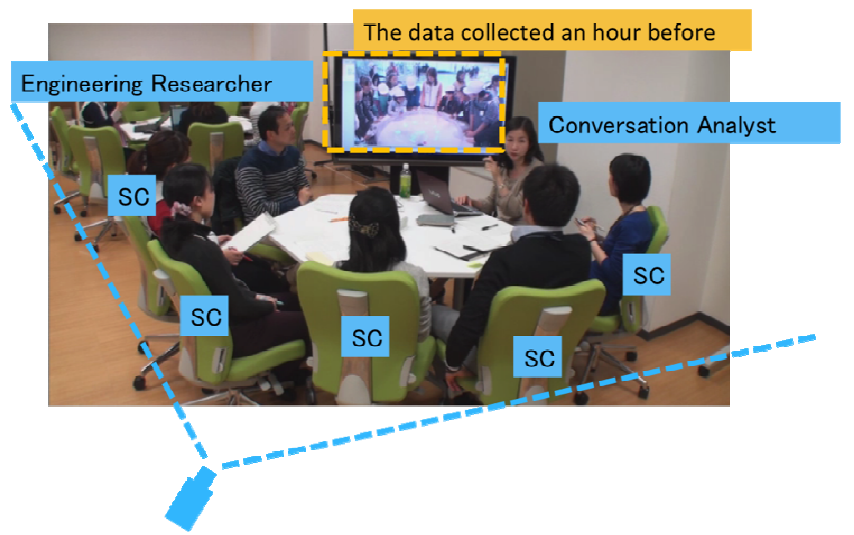

Fig. 2. Reflection meeting 

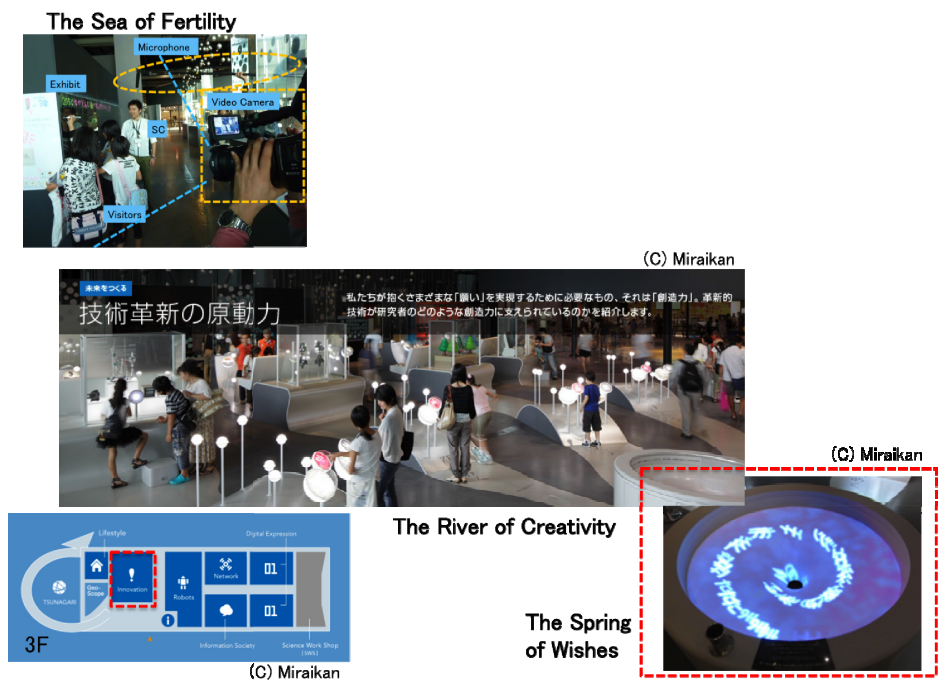

Fig. 3. Targeted Exhibition: 'The Driving Force of Innovation'

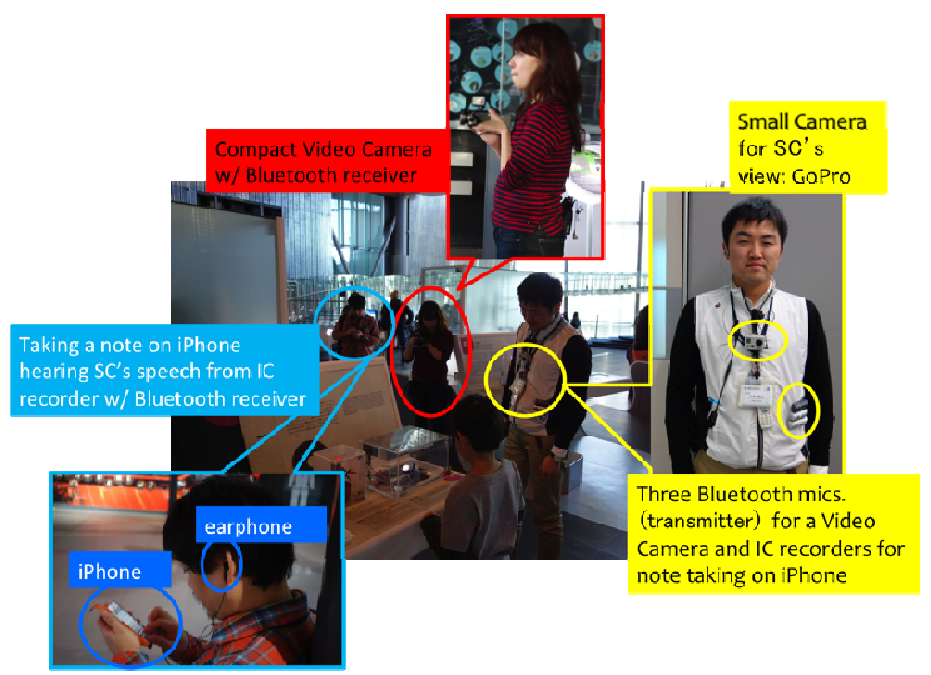

Fig. 4. Filming during the second phase

In the second phase, referring to our filming method, expert SCs filmed novice SCs' behaviors on the floor and reflection meetings between senior SCs and novice SCs for the purpose of training from February to September 2013. The devices used were basic home video cameras with a Bluetooth microphone for recording the SCs' speech. Three Bluetooth microphones were put on each SC's jacket, as shown in Figure 4; one 
was connected to a compact video camera, and the others were connected to earphones worn by conversation analysts who used iPhones to take notes.

In this paper, we use data filmed during the first phase.

\section{$4 \quad$ Analysis}

We show two kinds of data analysis: (1) multimodal analysis of naturally occurring science communication on the exhibition floor; and (2) findings from reflection meetings in which SCs and analysts observe the data analyzed in (1). In this case, because we had the reflection meeting a month after filming, we prepared a detailed transcript to introduce the basic concept of $\mathrm{CA}$ and the phenomena that happened there to SCs, including a targeted SC.

\section{1 'Who She Is' from Multimodal Data Analysis}

In this section, we introduce how an expert SC with a 7-year career initiated conversational sequences with junior high school students around the exhibition 'The Spring of Wishes' (see Fig. 3) and how she let them know who she was through several exchanges with them. Although she was wearing the SC uniform jacket, it seemed as though nobody noticed her in advance. Because the students are wearing identical white caps, they visited Miraikan on a school trip.

In our first observation, we found that visitors were unfamiliar with interacting with SCs, probably because the concept of SC was imported to Japan from overseas fairly recently. For instance, it seemed as though junior high school students, who visited the museum as part of a school trip to Tokyo, did not know about SCs in advance. In addition, however the alphabetical characters, 'science communicator', were printed on the back of SCs' jacket, it was too difficult for junior high school students to read and understand. To reveal details, we show multimodal analyses in this section. Thus, it is possible that most Japanese visitors do not notice a difference between themselves and SCs in terms of the amount of scientific knowledge they have.

Furthermore, it is possible that even SCs do not notice that visitors might not know what SCs are. To add details to this possibility, we show our observations of the impression made by an SC in section 4.2.

\section{Focus Point 1: How The Sc Initiates Conversational Sequences With Visitors.}

Unfortunately, we observed that SCs and visitors tended to talk about the purpose of filming and the camera operator at the beginning part of the first phase of filming. Because of this, we replaced the devices with simpler and smaller ones for the second phase of filming. However, we hoped that we would be able to observe a practical phenomenon: SCs employing the unusual atmosphere in which they are filmed by a high-definition camera as an environmental resource to initiate contact with visitors. 


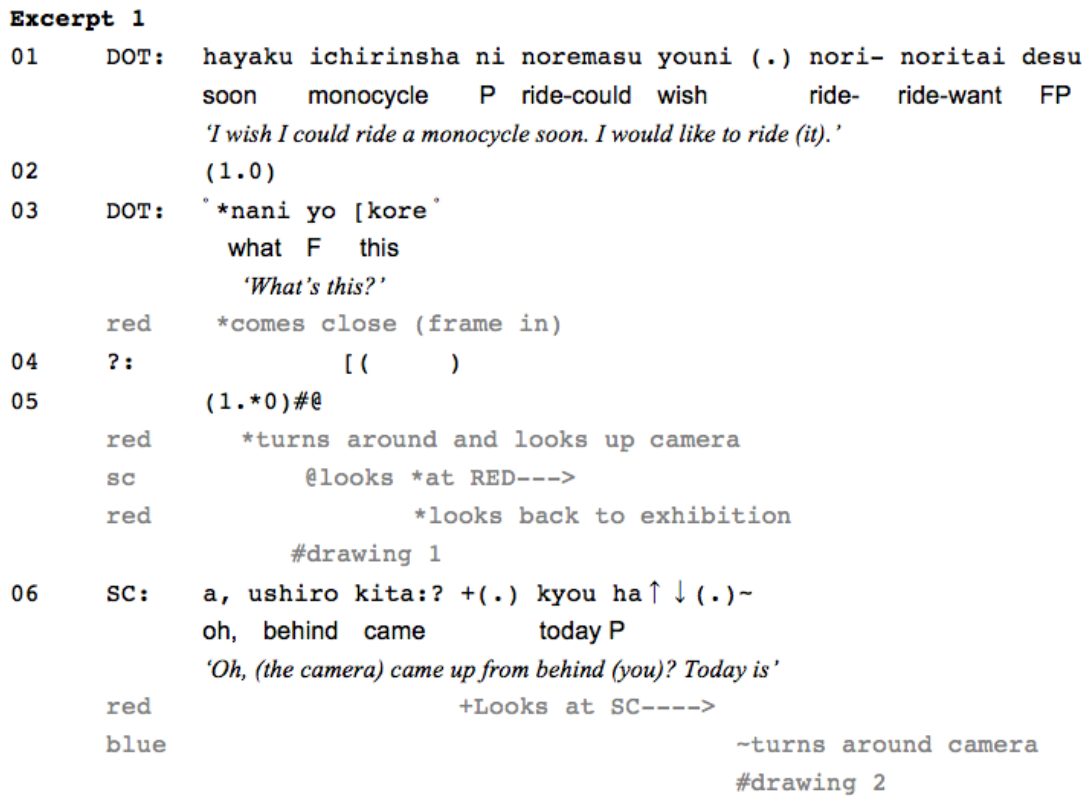

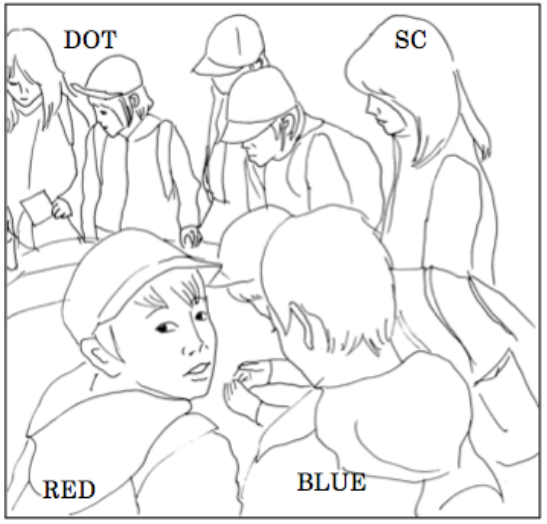

Drawing 1

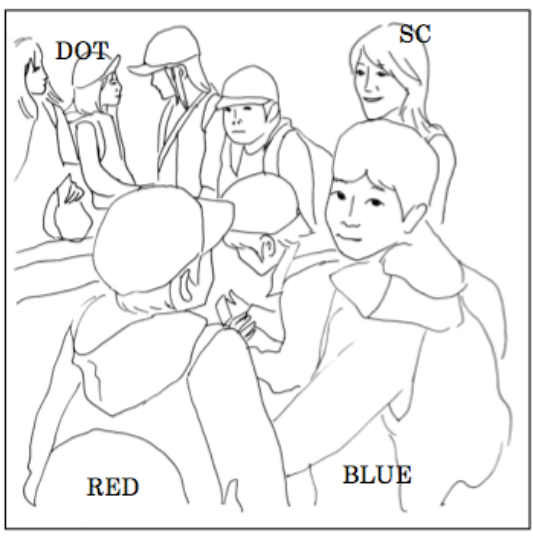

Drawing 2

Line 01 of Excerpt 1 is the beginning point of filming. The person labeled as DOT reads the letters that come up from the hall with a flat intonation. At this point, the SC has already taken a position in the frame touching the edge of the exhibition without getting the museum visitors' attention. She looks like she is listening to DOT's voice as well as those of the other visitors. In line 06, SC starts to talk with visitors, addressing to RED an 'a (oh)'-prefaced utterance indicating that she is changing her interactive state (Heritage, 1984); 'a, ushiro kita:?' 'Oh, (the camera) came up from behind (you)?' This utterance is a casual form of speech in Japanese, similar to talking with friends. 
RED comes closer to this gathering from outside the video frame at the beginning of line 03. Before that, he had already noticed that the cinematographer was filming them using a high-definition camera (see Drawing 1). Needless to say, the SC knew why the camera operator was filming them. We consider that SC tried to show the other participants that she had noticed RED's observing the camera operator by using an 'a (oh)'-prefaced utterance. As a result, SC got the attention of BLUE and that of the boy standing to the left of SC, and they changed the direction of their gaze toward the camera (see Drawing 2). In other words, she succeeded in inviting them into the participation framework between RED and SC.

It is striking that even though SC formulated her utterance as a question in line 06, SC continued her explanation about the filming activities of the cinematographer, 'kyou ha $\uparrow \downarrow$ (rising and falling intonations)' 'Today is,' without waiting for RED's reaction. So, despite the fact that 'a, ushiro kita:?' took the form of a question with rising intonation, it was not sequentially positioned as a first-pair-part (FPP) in this context (Schegloff \& Sacks, 1973). It seems that it worked as a device for getting the attention of other participants to let them hear her explanation.

We consider that her continuing to produce a next-turn constructional unit (TCU; Sacks, Schegloff, \& Jefferson, 1974) by rushing through the possible completion point and a transition relevance place (TRP) is an approach to forming a participation framework (Goffman, 1981), which is conducted at SC's initiative.

Thus, she initiates conversational sequences with visitors.

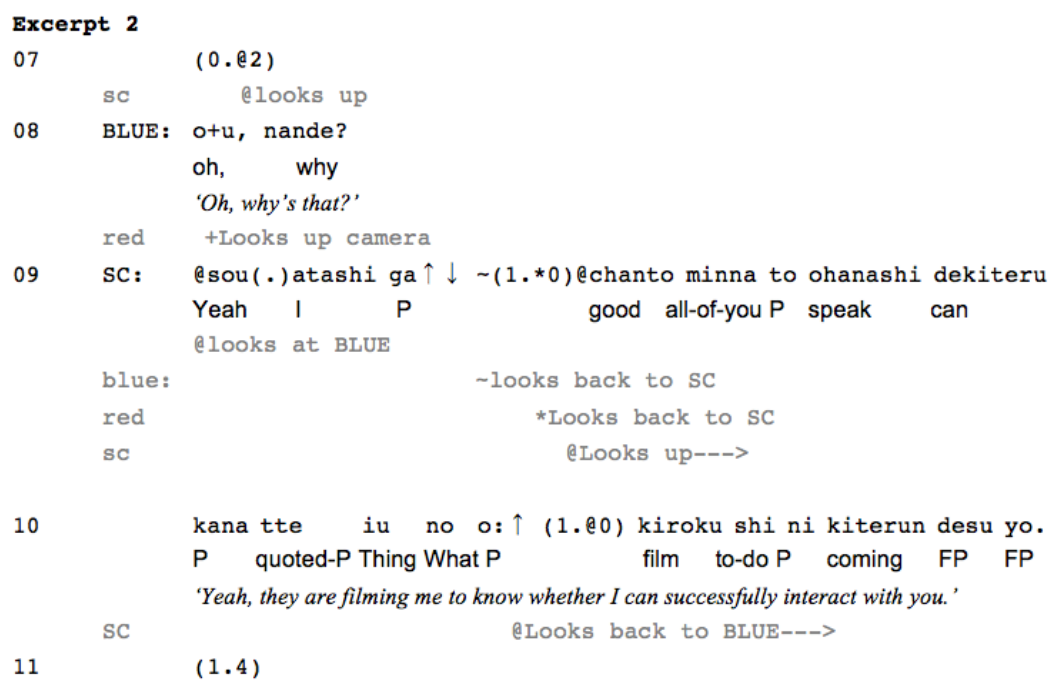

Focus Point 2: How She Let Them Know Who She Is. Excerpt 2 is a continuation of Excerpt 1. At the end of line 06, 'kyou ha $\uparrow \downarrow$ ', BLUE turns around to look for the camera, as shown in Drawing 2. Then, in line 08, BLUE says 'ou, nande?' 'Oh, why's that?' After SC answers BLUE saying 'sou' 'yeah' at the beginning of line 09, she restarts her explanation, which is formed by connecting it to her previous speech in line 06. 
Here, we would like to focus on why she did not say anything about 'who she is' using her job title, SC, at this moment. Instead, she just says, "chanto minna to ohanashi dekiteru kana te iu no o,' meaning 'whether I could successfully interact with you' in line 09 to line 10. In these lines, she represents the content of SCs' work, which is successfully interacting with visitors, in this case, to explain the reason that the camera operator was filming.

\author{
Excerpt 3 \\ 13 SC: datte miraikan tte kouiu hito ippai iru jan tatteru hito \\ because miraikan $Q$ like-these people many be FP standing people \\ 'You know, there are many people like me, who are standing (in front of exhibitions).'

We omitted one line between Excerpt 2 and Excerpt 3, in which DOT was reading the letters coming up from the hall. Like line 01 in Excerpt 1 and line 16 in Excerpt 3, this activity is not sequentially related to interactions between the SC and visitors. We would like to focus on why she, once again, did not say anything about 'who she is' using her job title directly. In line 13, she just said, 'datte miraikan tte kouiu hito ippai iru jan tatter hito,' 'You know, there are many people like me, who are standing (in front of exhibitions).' Especially, 'kouiu hito' 'LIKE-THESE PEOPLE,' 'people like me' and 'tatteru hito' 'STANDING PEOPLE,' 'people who are standing' are striking here. She mentioned two things, (1) the fact that there are a lot of people like her here, and (2) that they are standing near the exhibitions. (1) was designed as a confirmation question, i.e., 'jan' is a final particle used in Japanese to get confirmation from an interlocutor and (2) was incremental to the previous TCU. These examples make it seem as though she is trying to avoid saying her job title directly to visitors for some reason.

Finally in line 17, another visitor, STRIPE, asks her 'anaunsaa?' '(Are you) an announcer?' while coming close to the right side of the SC. Then, SC immediately answers his question in line 18, 'terebi janai \terebi janai' '(This is) not TV (station, this is) not TV (station).' 
Thus, she had never said who she is or what her position is called during the explanation of the reason for filming with high-definition camera. Because of that, visitors did not get crucial information about who she is at this moment.

Summury of Analyses and Discussions. In our analysis of Excerpt 1, we considered how the SC opens conversational sequences with visitors. We found that the SC utilized visitors' observations of the environmental resources surrounding them; a cameraman directed his camera to them. In our analysis of Excerpts 2 and 3, we investigated how she let them know who she is. We found that the SC never said her job title during the explanation of the reason for the camera operator's filming them. Instead, she mentioned the content of SC work, 'successfully interacting with you' (Excerpt 2) and the fact that there are a lot of people like her here, with brief mention of their characteristics (Excerpt 3). It seems that she tried to avoid saying her job title directly to visitors for some reason. We could not confirm why she did not say her job title at that moment or whether she intentionally formulated her explanation as we have suggested. However, we consider that if the SC used a job title to explain the situation, it is possible that nobody would understand the purpose of filming, i.e., visitors would not have the opportunity to learn any details concerning SCs' purpose, as in this case. In addition, their job title, 'science communicator', was imported into Japanese as a loan word using katakana, 'saiensu comyunikeetaa'. This Japanese convention of importing English words makes the practice of showing 'who I am' a complex matter in interaction.

\section{2 'Who I Am' from Practitioners' Voices in the Reflection Meeting}

In the reflection meeting, we showed some crucial points of our observations based on our analysis from the perspective of CA, as discussed in section 4.1, which involved watching the video clip repeatedly over the period of an hour. As a result, we obtained SCs' impressions of the issue of initiating conversational sequences and of whether the SC should always reveal who s/he is. One of the striking narratives by an SC in the reflection meeting was as follows:

I have never paid attention to whether visitors already knew who I was. On the other hand, it is possible for us to get visitors' information before interacting with them. Because the table on the working shift includes this information when we are going to have a group tour like a school trip. We always got it before interacting with them. Until now, we have been paying attention not to showing who we are but to knowing who they are, which means what are their interests, how much science knowledge they have, what they want to be in the future, and so on. However, I was very surprised there was the possibility that visitors did not know about the existence of SCs at Miraikan. This fact perhaps will be related to the planning of encounters with visitors in our future activities. 


\section{$5 \quad$ General Discussions and Conclusions}

As we mentioned in section 1, visiting a museum is an ambiguous event for not only visitors but also SCs from the perspective of territories of knowledge. In cases in which there is no common understanding between people about the amount of knowledge the other possesses, it might be difficult to understand who would provide new information for their knowledge to progress and to invite them to the activities at the museum. Of course, visitors may learn what an SC is through interacting with them directly. However, we suppose that if SCs know that most visitors do not know about the position or concept of SCs in advance, they could plan their interactions based on this condition.

This paper presents a preliminary result of our ongoing research project, focusing on the case of social encounters between SCs and visitors at a museum. When we design a museum guide robot, we tend to make it introduce itself at the beginning of interaction, such as 'Hi, my name is Nancy. I am a guide robot of this museum. Please ask me if you have any questions.' However, we found that this is not real in the case of museum where there is no explicit, pre-determined relationship regarding knowledge between SCs and visitors. The expert SC presented her role at the museum and the nature of her job through the interaction with the visitors. We consider that future technology should be designed integrating the insights gained from the analysis of our daily interactions.

Acknowledgments. This research was partially supported by The Center for Promotion of Integrated Scicences (CPIS), The Graduate University of Advanced Studies (SOKENDAI), Grant-in-Aid for Challenging Exploratory Research, Japan Society for the Promotion of Science, and Interaction Science Project: IDO-ROBO, Grand Challenge 2012-2013, National Institute of Informatics (NII).

\section{References}

1. Drew, P., Heritage, J.: Talk at Work: Interaction in Institutional Settings. Cambridge University Press, Cambridge (1992)

2. Goffman, E.: Forms of Talk. University of Pennsylvania Press, Philadelphia (1981)

3. Heath, C., Hindmarsh, J., Luff, P.: Video in Qualitative Research. Sage, London (2010)

4. Heritage, J.: A change-of-state token and aspects of its sequential placement. In: Atkinson, J.M., Heritage, J. (eds.) Structures of Social Action, pp. 299-345. Cambridge University Press, Cambridge (1984)

5. Heritage, J.: Epistemics in Action: Action Formation and Territories of Knowledge. Research on Language and Social Interaction 45, 1-29 (2012)

6. Sacks, H.: On the Analyzability of Stories by Children. In: Turner, R. (ed.) Ethnomethodology, pp. 216-232. Penguin, Harmondsworth (1974)

7. Sacks, H., Schegloff, E.A., Jefferson, G.: A simplest systematics for the organization of turn-taking for conversation. Language 50, 696-735 (1974)

8. Schegloff, E.A., Sacks, H.: Opening Up Closings. Semiotica VIII(4), 289-327 (1973) 